\title{
Health-Care Utilization and Complications of Endoscopic Esophageal Dilation in a National Population
}

\author{
Abhinav Goyal ${ }^{1}$, Kshitij Chatterjee ${ }^{2}$, Sujani Yadlapati ${ }^{1}$ and Shailender Singh ${ }^{3}$ \\ ${ }^{1}$ Department of Internal Medicine, Einstein Medical Center, Philadelphia, PA, ${ }^{2}$ University of Arkansas for Medical Sciences, Little Rock, AR, \\ ${ }^{3}$ Department of Gastroenterology, University of Nebraska Medical Center, Omaha, NE, USA
}

Background/Aims: Esophageal stricture is usually managed with outpatient endoscopic dilation. However, patients with food impaction or failure to thrive undergo inpatient dilation. Esophageal perforation is the most feared complication, and its risk in inpatient setting is unknown.

Methods: We used National Inpatient Sample (NIS) database for 2007-2013. International Classification of Diseases, 9th revision, Clinical Modification (ICD-9-CM) codes were used to identify patients with esophageal strictures. Logistic regression was used to assess association between hospital/patient characteristics and utilization of esophageal dilation.

Results: There were 591,187 hospitalizations involving esophageal stricture; $4.2 \%$ were malignant. Endoscopic dilation was performed in $28.7 \%$ cases. Dilation was more frequently utilized (odds ratio [OR], $1.36 ; p<0.001$ ), had higher in-hospital mortality $(3.1 \%$ vs. $1.4 \%$, $p<0.001$ ), and resulted in longer hospital stays ( 5 days vs. 4 days, $p=0.01$ ), among cases of malignant strictures. Esophageal perforation was more common in the malignant group $(0.9 \%$ vs. $0.5 \%, p=0.007)$. Patients with malignant compared to benign strictures undergoing dilation were more likely to require percutaneous endoscopic gastrostomy or jejunostomy (PEG/J) tube ( $14.1 \%$ vs. $4.5 \%, p<0.001)$. Palliative care services were utilized more frequently in malignant stricture cases not treated with dilation compared to those that were dilated.

Conclusions: Inpatient endoscopic dilation was utilized in $29 \%$ cases of esophageal stricture. Esophageal perforation, although infrequent, is more common in malignant strictures. Clin Endosc 2017;50:366-371

Key Words: Esophageal stenosis; Endoscopic dilation; Inpatients; Percutaneous endoscopic gastrostomy; Malignant stricture

\section{INTRODUCTION}

Esophageal stricture is an important cause of dysphagia in adults, and can result in considerable morbidity and mortality. Esophageal strictures can result from both benign and malignant processes. Benign strictures are most commonly related to gastroesophageal reflux disease followed by other etiologies such as eosinophilic esophagitis, radiation-induced,

Received: November 12, 2016 Revised: January 19, 2017

Accepted: January 19, 2017

Correspondence: Abhinav Goyal

Department of Internal Medicine, Einstein Medical Center, 5501 Old York Road, Suite 363 (Klein Building), Philadelphia, PA 19141, USA

Tel: +1-215-456-8520, Fax: +1-215-455-1933, E-mail: goyalabh@einstein.edu

(cc) This is an Open Access article distributed under the terms of the Creative Commons Attribution Non-Commercial License (http://creativecommons.org/ licenses/by-nc/3.0) which permits unrestricted non-commercial use, distribution, and reproduction in any medium, provided the original work is properly cited. postsurgical, or caustic ingestion. ${ }^{1,2}$ Malignant strictures, however, usually result from intrinsic processes such as esophageal carcinoma. $^{3}$

The goals of therapy for esophageal strictures are relief of dysphagia and prevention of stricture recurrence. ${ }^{4}$ Most cases of esophageal strictures are managed with elective outpatient endoscopic dilation. However, some patients present with food impaction or failure to thrive owing to severe dysphagia, undergo urgent inpatient endoscopic dilation. Esophageal perforation is the most feared complication of dilation, and its risk in this setting (i.e., urgent/inpatient setting) is not well described. Although the incidence of perforation is low, the mortality associated with dilation approaches $20 \%{ }^{5}$

The aim of this study was to explore the health-care utilization of endoscopic dilation for the treatment of esophageal strictures in the inpatient setting, and to define the rate of complications and outcomes of this procedure. 


\section{MATERIALS AND METHODS}

\section{Sample}

We used the National Inpatient Sample (NIS) database, which was constructed as part of the Healthcare Cost Utilization Project by the Agency of Healthcare Research and Quality for the years 2007 through $2013 .{ }^{6}$ NIS is the largest all-payer inpatient database in the United States. It is a $20 \%$ stratified sample of all discharges in a calendar year. Manufacturer-provided sampling weights were used to produce national estimates from the data.

International Classification of Diseases, 9th revision, Clinical Modification (ICD-9-CM) code 530.3 (stricture and stenosis of esophagus) was used to identify all patients with esophageal strictures. We then used ICD-9-CM codes 150.0, $150.1,150.2,150.3,150.4,150.5,150.8,150.9,230.1$, and V10.03 to identify patients with malignant strictures. Similarly, ICD9-CM procedure code 42.92 was used to identify patients who underwent endoscopic esophageal dilation during hospitalization. All patients aged $<18$ years, or with missing age or sex information were excluded from analysis.

\section{Statistical analysis}

The demographic characteristics and outcomes were calculated for all patients who underwent esophageal dilation for esophageal stricture and its subgroups (benign and malig-

Table 1. Demographic Characteristics and Outcomes among Patients with Esophageal Stricture Undergoing Endoscopic Dilation

\begin{tabular}{|c|c|c|c|c|}
\hline & Esophageal stricture & $\begin{array}{l}\text { Malignant stricture } \\
\text { subgroup }\end{array}$ & $\begin{array}{l}\text { Benign stricture } \\
\text { subgroup }\end{array}$ & $p$-value \\
\hline Number of observations (\% of total) & $169,618(100 \%)$ & $7,896(4.7 \%)$ & $161,722(95.3 \%)$ & \\
\hline Mean age in years (SD) & $70.4(15.4)$ & $67.8(11.5)$ & $70.5(15.5)$ & $<0.001$ \\
\hline Age categories ( $\%$ of total), yr & & & & $<0.001$ \\
\hline $18-39$ & 3.9 & 0.9 & 4.0 & \\
\hline $40-65$ & 28.2 & 37.9 & 27.8 & \\
\hline$>65$ & 67.9 & 61.2 & 68.2 & \\
\hline Sex (\% of total) & & & & $<0.001$ \\
\hline Male & 42.6 & 72.7 & 41.2 & \\
\hline Female & 57.4 & 27.3 & 58.8 & \\
\hline Race (\% of total) & & & & 0.01 \\
\hline Caucasian & 71.0 & 68.6 & 71.2 & \\
\hline African American & 9.5 & 10.9 & 9.5 & \\
\hline Others $^{\mathrm{a})}$ & 6.8 & 8.9 & 6.7 & \\
\hline Missing & 12.6 & 11.6 & 12.7 & \\
\hline Grouped Charlson index (\% of total) & & & & $<0.001$ \\
\hline 0 & 28.1 & 14.2 & 28.8 & \\
\hline 1 & 25.6 & 10.4 & 36.3 & \\
\hline 2 & 46.3 & 75.4 & 44.9 & \\
\hline \multicolumn{5}{|l|}{ Outcomes } \\
\hline Median length of stay (IQR), d & $4(3-8)$ & $5(3-9)$ & $4(3-8)$ & 0.01 \\
\hline Adjusted length of stay, $d$ & 4 & 4 & 4 & $\mathrm{~N} / \mathrm{A}$ \\
\hline Periprocedural mortality & 1.5 & 3.1 & 1.4 & $<0.001$ \\
\hline Esophageal perforation & 0.5 & 0.9 & 0.5 & 0.007 \\
\hline PEG/J placement & 4.9 & 14.1 & 4.5 & $<0.001$ \\
\hline
\end{tabular}

All the numbers reported in the table are in percentages unless otherwise specified.

SD, standard deviation; IQR, interquartile range; PEG/J, percutaneous endoscopic gastrostomy or jejunostomy; N/A, not available.

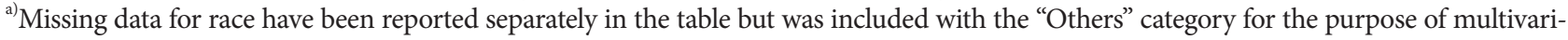
ate regression analysis. 
nant). The chi-square, Mann-Whitney, and independent-samples t-tests were used to compare for statistical significance. Multivariate logistic regression analysis was used to assess the impact of individual patient and hospital characteristics on the utilization of endoscopic dilation among patients with esophageal strictures. We used age groups (18-39, 40-64, and $\geq 65$ years), sex, race, and the Charlson comorbidity index for patient characteristics in the regression model. For hospital characteristics, we included the hospital bed size, hospital location (urban vs. rural), teaching status of the hospital, weekend versus weekday admission, and hospital region. The Charlson comorbidity index is a validated tool used for adjusting for comorbidities among databases based on ICD-9$\mathrm{CM}$ codes. We used a modification of the Charlson index to exclude the cancer comorbidity from the original index, given that esophageal cancer was an independent variable in our regression model.

A similar model was also used to assess for utilization of palliative care services among patients with malignant strictures. SPSS 23.0 (SPSS Inc., Chicago, IL, USA) and Stata 13.0 (StataCorp, College Town, TX, USA) were used for all statistical analyses. Institutional review board approval was not required as NIS is a de-identified national database.

\section{RESULTS}

Between 2007 and 2013, there were a total of 591,187 hospitalizations involving esophageal strictures, $4.2 \%$ of which were associated with esophageal malignancy (i.e., malignant stricture). Endoscopic dilation was performed in $28.7 \%$ cases of stricture. On subgroup analysis, however, endoscopic dilation was more frequently utilized in patients with malignant stricture than in patients with benign stricture stricture (31.9\% vs. $28.6 \%, p<0.001)$. Even after adjusting for various hospital and patient characteristics by using multivariate logistic regression analysis, endoscopic dilation remained more frequently utilized among patients with malignant stricture (odds ratio [OR], 1.36; confidence interval [CI], 1.27-1.46; $p<0.001$ ).

The demographic characteristics, hospital characteristics, and outcomes of patients with esophageal stricture who underwent endoscopic dilation are shown in Table 1. Men constituted a significant majority of patients with malignant stricture (72.7\%), in contrast to the benign stricture group (41.2\% male patients). Caucasians were the predominant racial group among both subgroups. Although most patients in both groups had a higher comorbidity burden (Charlson index 2), the malignant stricture subgroup had a significantly higher proportion in this category ( $75.4 \%$ vs. $44.9 \%, p<0.001)$.

The overall in-hospital or periprocedural mortality (mortality during the hospitalization when the procedure was performed was presumed to be directly or indirectly related to the procedure) was $1.5 \%$, and the median length of stay was 4 days. Patients with malignant strictures had a higher in-hospital mortality (3.1\% vs. $1.4 \%, p<0.001)$ and a longer hospital stay ( 5 days vs. 4 days, $p=0.01$ ). However, the length of stay for patients who were discharged alive to a non-facility location was 4 days for both groups. The incidence of esophageal per-

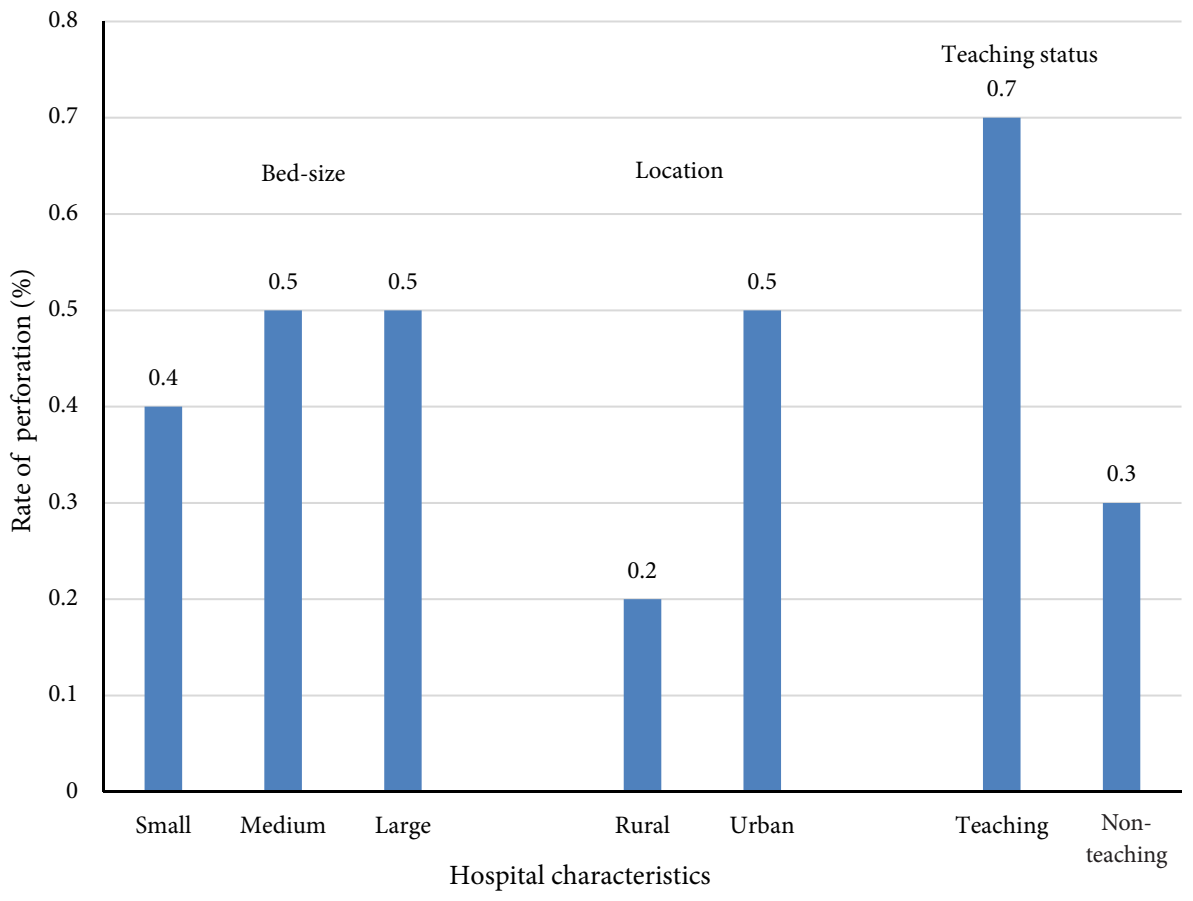

Fig. 1. Rate of esophageal perforation across different hospital characteristics. 
foration was very low in both groups, although it was slightly higher in the malignant group ( $0.9 \%$ vs. $0.5 \%, p=0.007)$. The rate of esophageal perforation according to various hospital characteristics is shown in Fig. 1. Patients with malignant stricture undergoing dilation were also significantly more likely to require percutaneous endoscopic gastrostomy or jejunostomy (PEG/J) tube placement ( $14.1 \%$ vs. $4.5 \%, p<0.001)$ during the same hospitalization compared with those with benign stricture. Hospital characteristics, PEG/J utilization, and palliative care utilization (encounter with the palliative care team) were also calculated for all patients with malignant stricture, including those not undergoing dilation, and are shown in Table 2. Overall, palliative care was utilized in 5.5\% cases of malignant stricture. It was utilized significantly more often in patients not undergoing stricture dilation $(6.6 \%$ vs. $3.1 \%, p<0.001)$. Even after adjusting for all patient and hospital characteristics by using multivariate regression analysis, as described above, palliative care remained significantly underutilized among those undergoing dilation compared with those who were not dilated (OR, 0.50 ; CI, $0.36-0.69 ; p<0.001$ ).

\section{DISCUSSION}

Esophageal dilation is a fairly common technique used for the treatment of benign and malignant esophageal strictures. However, its utilization in the inpatient setting is not well known. In our study, esophageal dilation was utilized in $28 \%$ of cases of esophageal stricture. Moreover, it was seen that strictures necessitating urgent/inpatient dilation were more likely to be malignant than benign. Piotet et al. demonstrated similar findings in their analysis of 2387 strictures in which $55 \%$ of the cases requiring dilation were malignant and $45 \%$ were benign. ${ }^{3}$ This finding is likely due to the rapid growth of malignant strictures and higher recurrence rate of symptoms necessitating repeated and urgent interventions.

The demographic characteristics of the subgroups showed that the malignant stricture group was slightly younger than the benign stricture group. This was likely a result of the high mortality of esophageal cancer, leading to a paucity of the older population and a lower mean age in this subgroup. Among male patients, there was a significant preponderance of malig-

Table 2. Hospital Characteristics and Utilization of Palliative Care Services and PEG/J among Patients with Malignant Esophageal Stricture

\begin{tabular}{|c|c|c|c|}
\hline & Dilation $(n=7,896)$ & No dilation $(n=16,789)$ & $p$-value \\
\hline Hospital teaching status & & & $<0.001$ \\
\hline Teaching & 38.3 & 45.8 & \\
\hline Nonteaching & 60.8 & 53.4 & \\
\hline Missing & 0.9 & 0.8 & \\
\hline Hospital location & & & 0.02 \\
\hline Rural & 6.7 & 8.9 & \\
\hline Urban & 92.4 & 90.3 & \\
\hline Missing & 0.9 & 0.8 & \\
\hline Hospital bed size & & & 0.96 \\
\hline Small & 9.8 & 10.2 & \\
\hline Medium & 22.3 & 21.8 & \\
\hline Large & 67.0 & 67.2 & \\
\hline Missing & 0.9 & 0.8 & \\
\hline Hospital region & & & 0.001 \\
\hline Northeast & 25.6 & 21.9 & \\
\hline Midwest & 24.8 & 22.7 & \\
\hline South & 33.6 & 35.4 & \\
\hline West & 16.0 & 20.0 & \\
\hline \multicolumn{4}{|l|}{ Utilization } \\
\hline PEG/J & 14.1 & 20.5 & $<0.001$ \\
\hline Palliative care services & 3.1 & 6.6 & $<0.001$ \\
\hline
\end{tabular}

All numbers in the table are percentages unless otherwise specified.

PEG/J, percutaneous endoscopic gastrostomy or jejunostomy. 
nant strictures requiring dilation. This is consistent with the higher prevalence of esophageal carcinoma in male patients reported in the literature.

Advances in endoscopic techniques and equipment have remarkably improved the safety of this procedure. The in-hospital mortality for patients undergoing dilation was relatively low (1.4\%). However, it was higher in the malignant stricture subgroup than in the benign stricture group. Similar trends in mortality were also reported by previous studies. ${ }^{3}$ Our findings also demonstrated a longer length of stay among patients with malignant stricture. However, to mitigate the effect of delay in discharge due to placement in skilled nursing or rehabilitation facilities, an adjusted length of stay was calculated in which only those patients who were discharged alive and to a nonfacility location were considered. This adjusted length of stay was the same for the two groups, thus pointing out that the apparent difference between the two groups might be a result of the higher proportion of patients with malignant stricture being discharged to facilities than home.

Although infrequent, perforation is the most dreaded complication of endoscopic dilation for an endoscopist, owing to the high mortality rate associated with the procedure. ${ }^{5}$ Previously published studies have shown perforation rates ranging from $0.1 \%$ to $5 \%{ }^{3.9-13}$ These estimates were mostly from elective esophageal dilations; however, our findings suggest that the incidence of esophageal perforation is exceedingly low even with inpatient semi-urgent dilation procedures. This may partly be because of the better dilation techniques being utilized nowadays, ${ }^{14,15}$ further reiterating the notion that the overall risk of esophageal perforation remains relatively low in patients undergoing dilation of esophageal stricture. Although rare, perforation seems to be more commonly encountered with dilation of malignant strictures $(0.9 \%$ vs. $0.5 \%)$. This trend is consistent with the existing literature on the subject. ${ }^{16,17}$ It is likely a result of the increased friability associated with previous use of chemotherapy or radiation therapy. Overall, patients with malignant strictures had worse outcomes than those with benign strictures.

When esophageal dilation is unsuccessful in providing symptom relief, the next step in management is usually to seek alternative forms of nutrition. Palliative care with or without PEG/J placement is another reasonable option for patients with malignant stricture. Our findings show that $4.9 \%$ of patients who undergo inpatient esophageal dilation require placement of a PEG/J tube during that hospitalization, likely owing to an unsuccessful procedure or procedural complication. It was also seen that PEG/J was far more commonly utilized in patients with malignant stricture (14.1\% vs. $4.5 \%)$, likely as a part of palliative care.

We also demonstrated that palliative care was utilized more often in patients with malignant stricture not undergoing dilation ( $6.6 \%$ vs. $3.1 \%)$. We conducted a multivariate logistic regression analysis to adjust for various patient-based and hospital-based confounders, such as the teaching status of the hospital, hospital location and region, bed size, weekend vs. weekday admission, age, race, sex, or other comorbidities. The odds of utilizing palliative care were twice as much if no dilation was performed. This may be because of the more advanced nature of cancer in this population. Palliative care in patients with esophageal cancer needs a multidisciplinary approach. Management of dysphagia involves endoscopic dilation and stenting; however, pain management is another crucial aspect, and palliative care physicians can be an essential part of the team in that regard. Although we found an in-

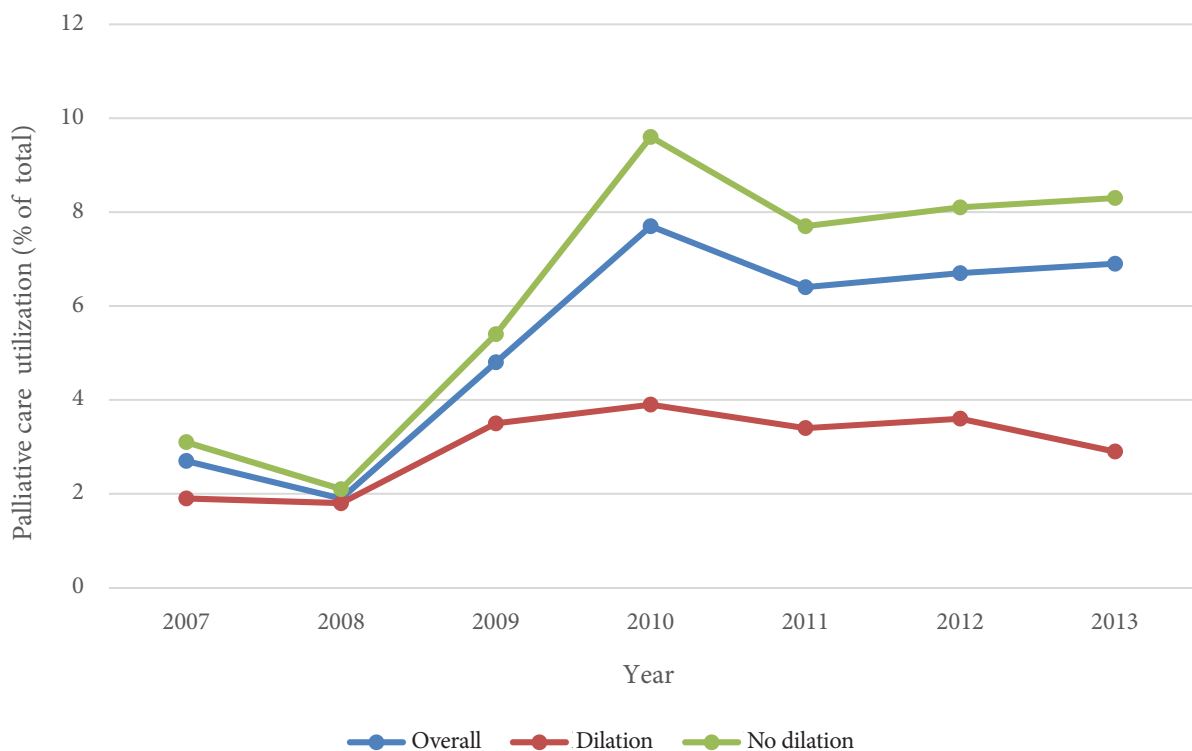

Fig. 2. Utilization of palliative care services among patients with malignant esophageal stricture. 
creasing trend from 2007 to 2013 (Fig. 2), our study revealed the striking underutilization of palliative care services in this patient population. ${ }^{18}$

Our study has some limitations. The proportion of patients presenting with acute food impaction or gradual failure to thrive among patients with malignant stricture is unclear. Moreover, information about the esophageal malignancy stage and treatment details such as prior chemo-radiation are not available, and these could have implications on the perforation rates. Furthermore, we could not account for patients undergoing repeat dilations.

In conclusion, dilation of esophageal strictures is commonly used in the hospital inpatient setting and is relatively safe. Although complications, particularly perforations, are rare, patients need to be carefully monitored and aggressively treated when perforations do occur. Patients with malignant esophageal strictures undergoing esophageal dilation have worse outcomes than patients with benign strictures.

\section{Conflicts of Interest}

The authors have no financial conflicts of interest.

\section{REFERENCES}

1. ASGE Standards of Practice Committee, Pasha SF, Acosta RD, et al. The role of endoscopy in the evaluation and management of dysphagia. Gastrointest Endosc 2014;79:191-201.

2. Richter JE. Peptic strictures of the esophagus. Gastroenterol Clin North Am 1999;28:875-891, vi.

3. Piotet E, Escher A, Monnier P. Esophageal and pharyngeal strictures: report on 1,862 endoscopic dilatations using the Savary-Gilliard technique. Eur Arch Otorhinolaryngol 2008;265:357-364.
4. Standards of Practice Committee, Egan JV, Baron TH, et al. Esophageal dilation. Gastrointest Endosc 2006;63:755-760.

5. Brinster CJ, Singhal S, Lee L, Marshall MB, Kaiser LR, Kucharczuk JC. Evolving options in the management of esophageal perforation. Ann Thorac Surg 2004;77:1475-1483

6. AHRQ. NIS overview [Internet]. Rockville (MD): Healthcare cost and utilization project (HCUP); c2016 [updated 2016 Dec 14; cited 2016 Dec 15]. Available from: https://www.hcup-us.ahrq.gov/nisoverview.jsp.

7. Zhang Y. Epidemiology of esophageal cancer. World J Gastroenterol 2013;19:5598-5606.

8. Shami VM. Endoscopic management of esophageal strictures. Gastroenterol Hepatol (N Y) 2014;10:389-391.

9. Fry LC, Mönkemüller K, Neumann H, Schulz HU, Malfertheiner P. Incidence, clinical management and outcomes of esophageal perforations after endoscopic dilatation. Z Gastroenterol 2007;45:1180-1184.

10. Hernandez LV, Jacobson JW, Harris MS. Comparison among the perforation rates of Maloney, balloon, and savary dilation of esophageal strictures. Gastrointest Endosc 2000;51(4 Pt 1):460-462.

11. Jethwa P, Lala A, Powell J, McConkey CC, Gillison EW, Spychal RT. A regional audit of iatrogenic perforation of tumours of the oesophagus and cardia. Aliment Pharmacol Ther 2005;21:479-484.

12. Pereira-Lima JC, Ramires RP, Zamin I Jr, Cassal AP, Marroni CA, Mattos AA. Endoscopic dilation of benign esophageal strictures: report on 1043 procedures. Am J Gastroenterol 1999;94:1497-1501.

13. Silvis SE, Nebel O, Rogers G, Sugawa C, Mandelstam P. Endoscopic complications. Results of the 1974 American society for gastrointestinal endoscopy survey. JAMA 1976;235:928-930.

14. Riley SA, Attwood SE. Guidelines on the use of oesophageal dilatation in clinical practice. Gut 2004;53 Suppl 1:i1-i6.

15. Langdon DF. The rule of three in esophageal dilation. Gastrointest Endosc 1997;45:111.

16. Hagel AF, Naegel A, Dauth W, et al. Perforation during esophageal dilatation: a 10-year experience. J Gastrointestin Liver Dis 2013;22:385-389.

17. Mocanu A, Bârla R, Hoara P, Constantinoiu S. Endoscopic palliation of advanced esophageal cancer. J Med Life 2015;8:193-201.

18. The American cancer society medical and editorial content team. Palliative therapy for cancer of the esophagus [Internet]. Atlanta (GA): American cancer society; c2014 [updated 2016 Feb 4; cited 2016 Dec 15]. Available from: https://www.cancer.org/cancer/esophagus-cancer/ treating/palliative-therapy.html. 